\title{
Urinary excretion of 2-methyl-2,3-butanediol and 2,3-pentanediol in patients with disorders of propionate and methylmalonate metabolism
}

\author{
M. Duran ${ }^{\text {a }}$, D. Ketting a ${ }^{\text {, L. Bruinvis }}{ }^{\text {a }}$, L. Dorland ${ }^{\text {a }}$, S.K. Wadman ${ }^{\text {a }}$ \\ and J.A.J.M. Bakkeren ${ }^{b}$ \\ a University Children's Hospital 'Het Wilhelmina Kinderziekenhuss', Utrecht and ${ }^{b}$ University of Nijmegen, \\ Department of Paediatrics, Nijmegen (The Netherlands)
}

Key words: Propionic acidemia; Methylmalonic acidemia; Urinary diols; 2-Methyl-2,3-butanediol; 2,3-Pentanediol

\section{Summary}

Urine samples from patients with propionic acidemia and from a patient with methylmalonic acidemia contained unknown non-acidic metabolites by gas chromatography/mass spectrometry after ethyl acetate extraction. It could be demonstrated by mass spectrometric studies and by synthesis of reference compounds that the major metabolite was 2-methyl-2,3-butanediol, while smaller amounts of 2,3pentanediol were also present. These diols were present in abnormal amounts in these patients during attacks of metabolic decompensation.

\section{Introduction}

Patients with propionic acidemia (PA), methylmalonic acidemia (MMA) and multiple carboxylase deficiency (MCD) have been shown to excrete various characteristic abnormal metabolites derived from propionyl-CoA. Substances of major diagnostic importance are 3-hydroxypropionate and methylcitrate [1], whereas very high concentrations of propionate-derived ketone bodies such as 3-hydroxy$n$-valerate can be found during episodes of metabolic decompensation [2]. The formation of several of these metabolites occurs as a result of competition of propionyl-CoA with acetyl-CoA as substrates for certain enzyme reactions such as these catalysed by citrate synthase and acetoacetyl-CoA thiolase. Other reactions

Correspondence to: M. Duran, University Children's Hospital 'Het Wilhelmina Kinderziekenhuis', Nieuwe Gracht 137, NL 3512 LK Utrecht, The Netherlands. 
are directed towards the sequestration of coenzyme A, exemplified by the formation of propionyl carnitine [3] and propionylglycine. Nearly all propionate metabolites have an acidic character; consequently they can be detected by the analysis of urinary organic acids. Butanone, the (non-)enzymatically formed degradation product of 3-oxo- $n$-valerate and of 2-methyl-3-oxobutyrate [4] is one of the few exceptions.

In this report, we describe the identification of two non-acidic metabolites, viz. 2-methyl-2,3-butanediol and 2,3-pentanediol, which have not previously been described in man. Correlations with the excretion levels of other metabolites are tentatively given while their formation from presently unknown precursors will be discussed.

\section{Materials and methods}

The $\mathrm{C}_{5}$-diols that could be obtained commercially were purchased from the following sources; 1,4-pentanediol from Aldrich-Chemie (Steinheim, FRG); 1,5pentanediol from Fluka (Buchs, Switzerland); 2,4-pentanediol from Aldrich-Chemie. Several pentanediols were synthesized by lithium aluminium hydride reduction of parent compounds: 1,3-pentanediol was synthesized from 3-oxovaleric acid; 3methyl-1,3-butanediol from 3-hydroxyisovaleric acid which had been synthesized in our laboratory previously and 2-ethyl-1,3-propanediol from ethylmalonic acid. 2,3-Pentanediol was obtained by reduction of 2,3-pentanedione (Aldrich-Chemie) with sodium borohydride.

The target compound 2-methyl-2,3-butanediol was synthesized from 2-methyl-3buten-2-ol (Aldrich-Chemie) by reduction after oxymercuration [5]. Mercuric acetate $(0.1 \mathrm{~mol})$ was dissolved in $100 \mathrm{ml} \mathrm{H}_{2} \mathrm{O}$, followed by the rapid addition of $100 \mathrm{ml}$ tetrahydrofuran. The orange-yellow suspension was stirred for $15 \mathrm{~min}$ and subsequently $0.1 \mathrm{~mol}$ of 2-methyl-3-buten-2-ol was added, upon which discolouration took place. Stirring for another hour was followed by the subsequent addition of $100 \mathrm{ml} 3 \mathrm{~mol} / 1 \mathrm{NaOH}$ and $50 \mathrm{mmol} / 1 \mathrm{NaBH}_{4}$ in $100 \mathrm{ml} 3 \mathrm{~mol} / 1 \mathrm{NaOH}$. The temperature of the reaction mixture was maintained at $25^{\circ} \mathrm{C}$. Free mercury was formed during the process. Finally the mixture was stirred vigorously at room temperature and left standing overnight.

Short-chain diols were analysed by gas chromatography of their trimethylsilyl ethers after extraction from the urine with ethyl acetate as described before [6]. The percent recovery of the diols improved with increasing chain length: for 1,2-propanediol a value of $12 \%$ was calculated, whereas $21.5 \%$ of 2,3 -butanediol and $44 \%$ of 1,5-pentanediol were recovered respectively.

Both electron impact and chemical ionisation mass spectrometry $\mathrm{NH}_{3}$ as the reactant gas) were used for identification. The ionizing voltage and the pressure of the reactant gas were identical to the values described before [6].

\section{Patients}

Six patients were included in this study, five with propionic acidemia (PA) and one with methylmalonic acidemia (MMA). The latter suffered from a methyl- 
malonyl-CoA mutase deficiency. All patients with propionic acidemia in this study were admitted for severe metabolic acidosis in the first week(s) of life. Propionic acidemia is one of the most life-threatening inborn errors of metabolism; four of the five affected patients died very early in life. The index patient is currently $19 \mathrm{mth}$ old; his dietary 'protein' consists of an amino acid mixture restricted in valine, isoleucine, methionine, and threonine (Milupa OS).

\section{Results}

The diagnosis of methylmalonic acidemia or propionic acidemia is usually made by the analysis of urinary organic acids. A large amount of methylmalonic acid generally dominates the profile in MMA-patients, whereas the profile observed in PA-patients can be variable. A characteristic organic acid pattern of a PA-patient is shown in Fig. 1. The patient was a very sick neonate who suffered from an extreme ketoacidosis, as is evident from the high excretion of 3-hydroxybutyrate (peak 7). Methylcitrate isomers (peak 10) and 3-hydroxypropionate (peak 6) together with the alternative ketone body 3-hydroxyvalerate (peak 8 ) were the main propionyl-CoArelated organic acids. In addition, moderate concentrations of various other characteristic metabolites such as 2-methyl-3-hydroxybutyrate, 3-oxovalerate, 2-methyl3-hydroxyvalerate, propionylglycine, tiglylglycine, 4-methylpimelate, 4-methylsub-

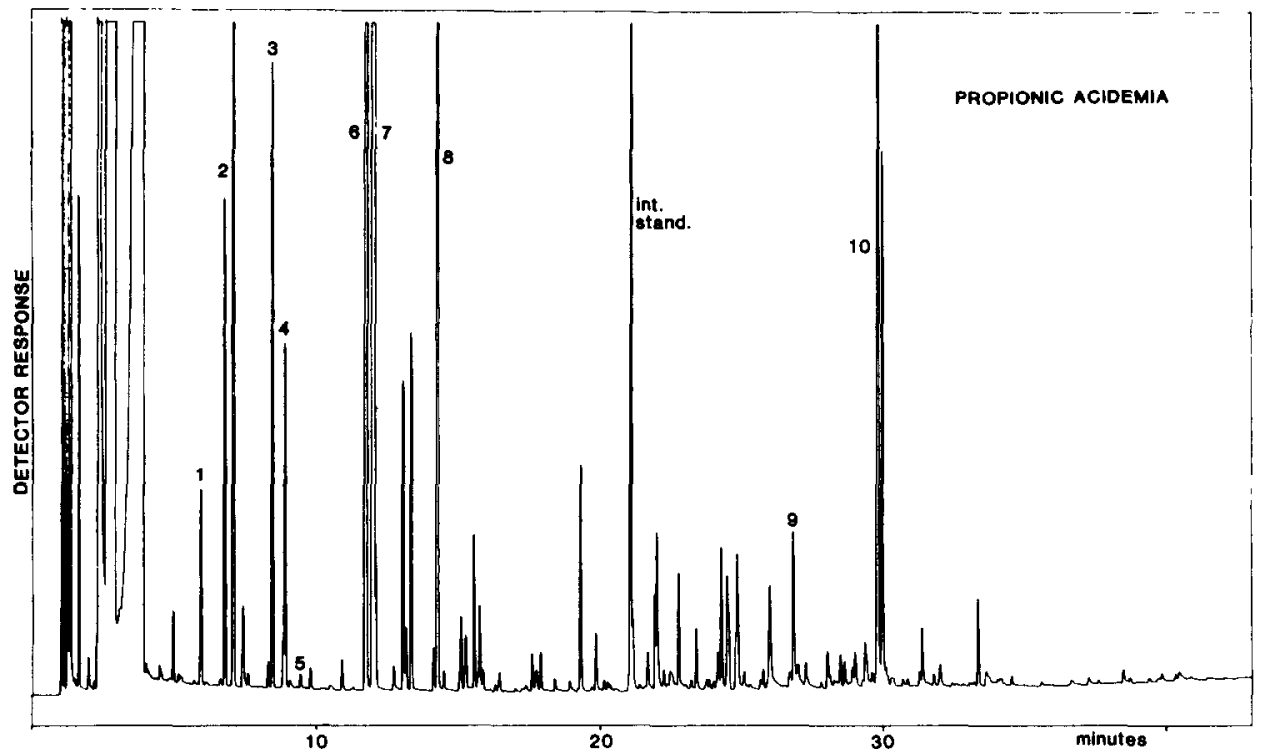

Fig. 1. Gas chromatogram of urinary ethyl acetate extractable metabolites in a patient with propionic acidemia (TMS esters or ethers). The following substances are indicated: 1, 1,2-propanediol; 2, 2,3butanediol (two peaks); 3, 2-methyl-2,3-butanediol; 4, lactic acid; 5, 2,3-pentanediol; 6, 3-hydroxypropionic acid; 7, 3-hydroxybutyric acid; 8, 3-hydroxyvaleric acid; $9, \mathrm{~N}$-tiglylglycine; 10 , methylcitric acid (two peaks); int. stand., 3-phenylbutyric acid. 


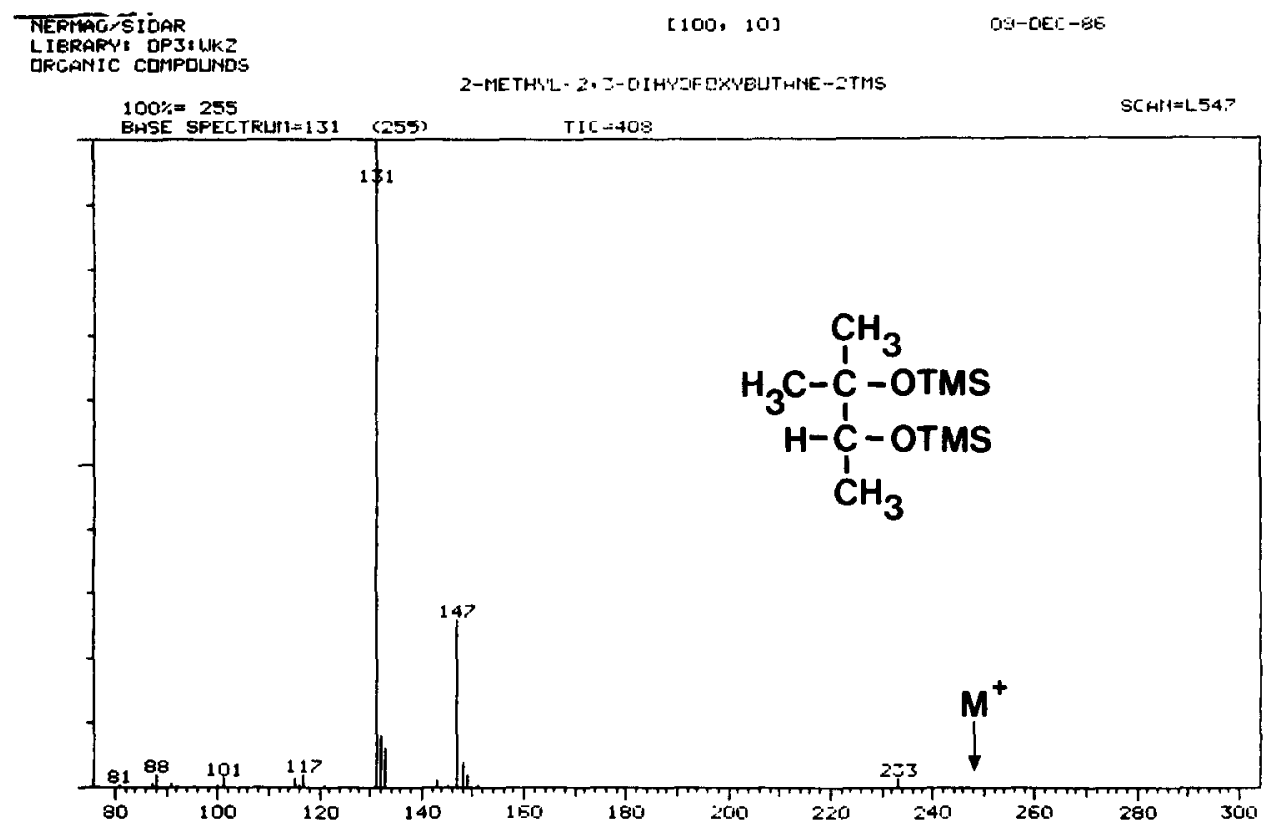

Fig. 2. Electron impact mass spectrum of the di-TMS ether of synthetic 2-methyl-2,3-butanediol. The fragmentation pattern was identical to that observed for the urinary component.

erate, and 2-methylglutaconate were found. The first part of the chromatogram showed several large peaks of volatile compounds, among which 1,2-propanediol (peak 1) and 2,3-butanediol (peaks 2). Electron impact mass spectrometry of the compound generating peak 3 is shown in Fig. 2. Its apparent molecular mass was 248 and the fragment at $m / z=147$ indicated that the molecule contained two TMS-groups. Its GC retention time - the compound eluted just before lactate (Fig. 1) - ruled out the possibility of a hydroxybutanoic acid. Comparison of the spectrum and the retention time with a number of different dihydroxypentanes showed 2-methyl-2,3-butanediol to be the most likely structure. The reference compound, synthesized by hydration of the corresponding unsaturated branched pentanol, appeared to behave identically to the urinary product. The mass spectrum of 2-methyl-2,3-dihydroxybutane was dominated by the fragment at $m / z=131$, representing $\left[\left(\mathrm{CH}_{3}\right)_{2}-\mathrm{C}-\mathrm{OTMS}\right]^{+}$. Surprisingly the abundance of the second expected fragment at $m / z 117$ was negligible. Although the molecular ion appeared to be absent, amplification of the abundance in the higher mass range combined with application of chemical ionization mass spectrometry pinpointed it at 248 .

Our finding of this diol prompted us to search for related substances. Subsequently, small amounts of 2,3-pentanediol were discovered (Fig. 1, peak 5). Its EI-mass spectrum showed marked similarities to the branched analogue, the difference lying in the relative abundance of the fragment at $m / z-117$ (Fig. 3). Again the molecular ion was quasi absent. 


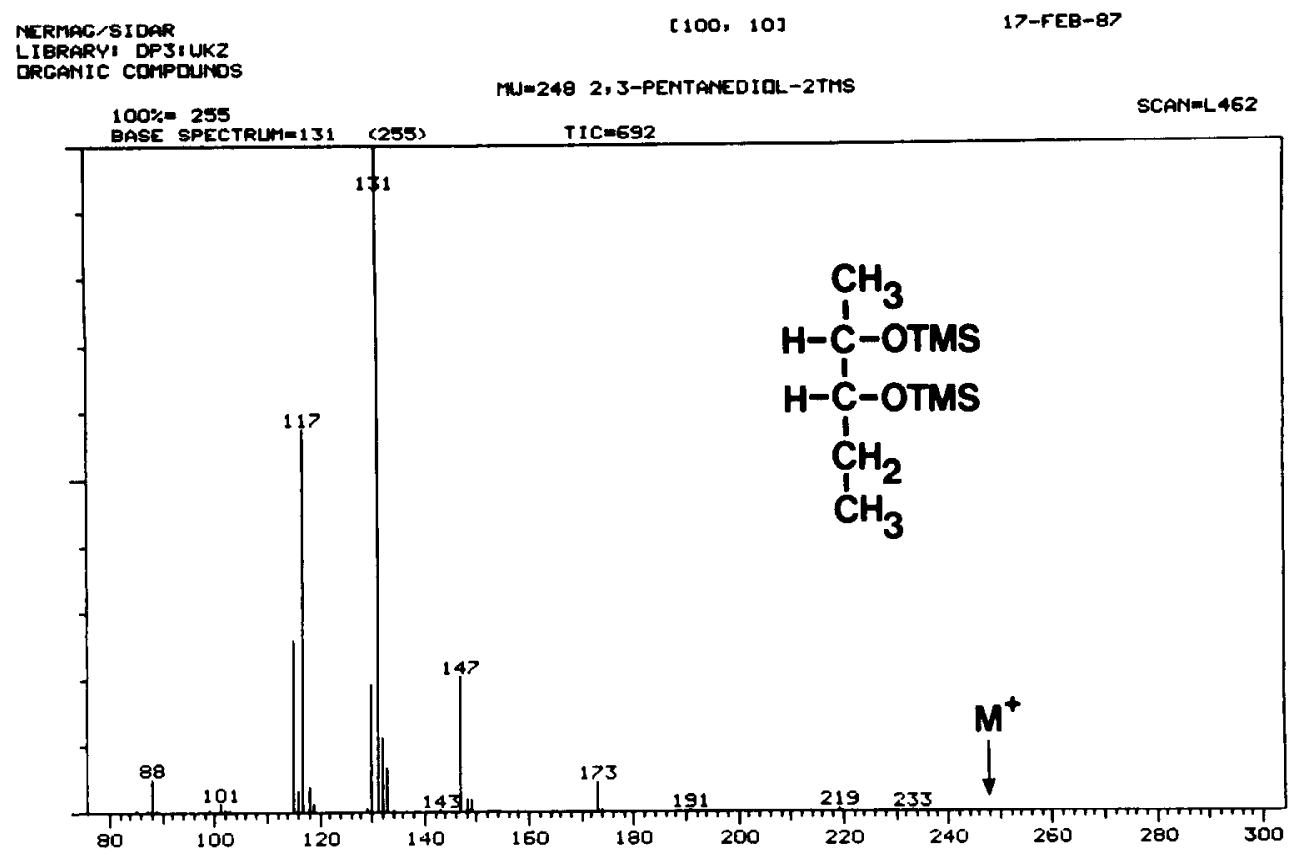

Fig. 3. Electron impact mass spectrum of the di-TMS ether of synthetic 2,3-pentanediol. Fragmentation proceeded analogous to the urinary substance.

All patients excreting 2-methyl-2,3-butanediol and 2,3-pentanediol also displayed markedly increased urinary levels of 1,2-propanediol and 2,3-butanediol (Table I). However, there was no apparent linear or inverse relationship between the excretion levels of the $\mathrm{C}_{3^{-}}, \mathrm{C}_{4^{-}}$, and $\mathrm{C}_{5}$-diols. Although the urine samples that we tested were random samples, all patients were in a state of severe metabolic decompensation at the time of collection. Four of the six patients were ketoacidotic, as demonstrated by the increased urinary excretion of 3-hydroxybutyrate (data not shown). Accordingly these patients also excreted propionyl-CoA derived products of ketogenesis such as 3-hydroxyvalerate and 2-methyl-3-hydroxyvalerate.

\section{TABLE I}

Urinary excrction of short-chain diols in patients with propionic acidemia and methylmalonic acidemia ${ }^{\text {a }}$

\begin{tabular}{lllccc}
\hline Patient & Diagnosis & $\begin{array}{l}\text { 1,2-Propane- } \\
\text { diol }\end{array}$ & $\begin{array}{l}2,3-\text { Butane- } \\
\text { diol }\end{array}$ & $\begin{array}{l}2 \text { - } \mathrm{CH}_{3} \text {-2,3-bu- } \\
\text { tanediol }\end{array}$ & $\begin{array}{c}2,3 \text {-Pen- } \\
\text { tanediol }\end{array}$ \\
\hline 1 & PA & 1130 & 3093 & 871 & 24 \\
2 & PA & 990 & 3026 & 648 & 26 \\
3 & PA & 550 & 1634 & 317 & tr \\
4 & PA & 441 & 12007 & 547 & 143 \\
5 & PA & 248 & 392 & 249 & 23 \\
6 & MMA & 9669 & 2921 & 1253 & 26 \\
\hline
\end{tabular}

a Concentrations are given in $\mu \mathrm{mol} / 1$. 
All patients, thus including the one with methylmalonic acidemia, excreted large amounts of methylcitrate and 3-hydroxypropionate, the clearest sign of propionylCoA accumulation. The intermediary metabolites of the isoleucine catabolic pathway such as 2-methyl-3-hydroxybutyrate and tiglylglycine were present in some, but not all patients.

A patient wich beta-ketothiolase deficiency, who was admitted during an attack of severe ketoacidosis, did not excrete the here described pentanediols, nor did he excrete appreciable amounts of 1,2-propanediol or 2,3-butanediol. Also the urine samples from a patient with biotinidase deficiency were found to be free of short-chain diols.

Short-chain diols have one or two asymmetric centres. We are currently investigating the possibilities to establish the absolute configuration of the urinary pentanediols in PA and MMA patients. The fact that 2,3-butanediol was eluted as two peaks (Fig. 1) in the present patients implicates that both the $R R$ and the meso-form were probably excreted. Inhomogeneity in the small peak representing 2,3-pentanediol was not observed.

\section{Discussion}

When propionic acidemia was discovered nearly 20 years ago, the diagnosis was made by finding an abnormally high plasma propionate level [7]. As the analysis of plasma volatile fatty acids is a cumbersome technique, many researchers have looked for characteristic urinary metabolites which allowed a reliable and fast diagnosis $[1,8]$. To date at least 17 metabolites have been detected. The relative importance of each metabolite is not to be discussed here. It is well known that the detection of any characteristic metabolite may be evidence for the presence of a defect in propionate metabolism.

2-methyl-2,3-Butanediol and 2,3-pentanediol are metabolites which have not previously been described in man. These substances most likely reflect the accumulation of propionyl-CoA, because they were only found in the urines of patients with propionic and with methylmalonic acidemia. Although this would permit their use in the diagnosis of these disorders, they do not have advantages over the use of other metabolites such as methylcitrate and 2-methyl-3-hydroxy(or oxo)valerate for this purpose. Moreover, in our experience the diols were only present in severely decompensated patients, while methylcitrate is virtually always present in PA patients [1] and methylmalonic acid is the biochemical hallmark of MMA patients.

The mode of formation of these newly identified metabolites is of interest, but not easily explained. It is attractive to try and find parallels between the ways of formation of butanediols and pentanediols. Elevated concentrations of butanediol have been associated with excessive alcohol intake [9] and with neonatal metabolic acidosis [10]. Several investigators have speculated on endogenous formation of 2,3-butanediol from ethanol in alcoholics [11], although an exogenous formation of the diol as a product of intestinal fermentation could not be ruled out completely, Hommes et al [10] put forward the hypothesis that 2,3-butanediol could be formed from pyruvate and acetyl-CoA via acetolactate by bacteria. By a similar mechanism 
2,3-pentanediol could be formed from acetyl-CoA and 2-oxo-butyrate, a known precursor of propionyl-CoA. More difficult to explain is the formation of the major metabolite 2-methyl-2,3-butanediol.

However, when methylcitrate is metabolized in the citric acid cycle, a number of candidate precursor molecules such as mesaconate, citramalate, and oxalopropionate are formed. These substances have the correct backbone for the formation of 2-methyl-2,3-butanediol. There is only one indication for this type of methylcitrate metabolism, viz. the detection of citramalate in the urine of propionic acidemia patients [12]. It is not feasible to test this hypothetical metabolic pathway, because methylcitrate is not commercially available as far as we know. Hence loading tests are impossible. Several reports have been published on the formation of short-chain diols (1,2-propanediol and 2,3-butanediol) from acetone in rats [13,14]. However, none of these publications contains valid metabolic schemes describing the formation of $\mathrm{C}_{4}$-diols from acetone. We do not have the impression that ketotic patients excrete abnormal amounts of butanediol. In man the propanediols are not likely of endogenous origin, in view of their use as a solvent for drugs. Attractive as it would be to link the formation of the two pentanediols to the occurrence of 2-butanone [4], we consider it difficult to describe a simple relation between the two types of compounds. Against the role of 2-butanone as the precursor molecule is the fact that two of our patients were not ketotic at the time of the study and thus were unlikely to accumulate this ketone.

Endogenous or exogenous formation, this problem has not yet been solved. It has been known for some time that patients with a resection of part of the small bowel excrete 3-hydroxypropionate [15], probably produced from propionate by the intestinal flora. We investigated similar patients, but could not find an abnormal excretion of short-chain diols. The best way to solve the question of the site of 2-methyl-2,3-butanediol production might be the analysis of faeces from a PA or MMA patient. Unfortunately, this material was not available.

The biological significance of the occurrence of short-chain diols in patients with organic acidemia is an open question. We even do not know if a compound such as 2-methyl-2,3-butanediol is metabolized further (e.g. by alcohol dehydrogenase) or if it is to be regarded as an end product. Further studies will be needed to elucidate whether short-chain diols play a role in the development of episodes of metabolic decompensation in these patients. These studies might give more insight into the link between fat and carbohydrate metabolism as a means of energy conservation [16].

\section{References}

1 Duran M, Gompertz D, Bruinvis L, Ketting D, Wadman SK. The variability of metabolite excretion in propionic acidaemia. Clin Chim Acta 1978;82:93-99.

2 Truscott RJW, Pullin C, Halpern B, Hammond J, Haan E, Danks DM. The identification of 3-keto-2-methylvaleric acid and 3-hydroxy-2-methylvaleric acid in a patient with propionic acidemia. Biomed Mass Spectrom 1979;6:294-300.

3 Chalmers RA, Roe CR, Stacey TE, Hoppel CL. Urinary excretion of L-carnitine and acylcarnitines by patients with disorders of organic acid metabolism: evidence for secondary insufficiency of $\mathbf{L}$-carnitine. Pediat Res 1984;18:1325-1328. 
4 Menkes JH. Idiopathic hyperglycinemia: isolation and identification of three previously undescribed urinary ketones. J Pediat 1966;69:413-421.

5 Furniss BS, Hannaford AJ, Rogers V, Smith PWG, Tatchell AR. Vogel's textbook of practical organic chemistry, ed. 4. London:Longman, 1978;378.

6 Duran M, Ketting D, Van Vossen R, Beckeringh TE, Dorland L, Bruinvis L, Wadman SK. Octanoylglucuronide excretion in patients with a defective oxidation of medium-chain fatty acids. Clin Chim Acta 1985;152:253-260.

7 Hommes FA, Kuipers JRG, Elema JD, Jansen JF, Jonxis JHP. Propionicacidemia, a new inborn error of metabolism. Pediat Res 1968;2:519-524.

8 Jakobs C, Dorland L, Sweetman L, Duran M, Nyhan WL, Wadman SK. Identification of methylbranched chain dicarboxylic acids in amniotic fluid and urine in propionic and methylmalonic acidemia. Pediat Res 1984;18:1185-1191.

9 Sisfontes L, Nyborg G, Jones AW, Blomstrand R. Occurrence of short-chain aliphatic diols in human blood: identification by gas chromatography-mass spectrometry. Clin Chim Acta 1986;155:117-122.

10 Hommes FA, Bruins AP, Dajani-Wielaard J, Baarsma R. 2,3-Dihydroxybutane: an unusual compound found in the gaschromatographic analysis of volatile compounds of urine. J Clin Chem Clin Biochem 1979;17:97-99.

11 Rutstein DD, Nickerson RJ, Vernon AA, Kishore P, Veech RL, Felver ME, Needham LL, Thacker SB. 2,3-Butanediol: an unusual metabolite in the serum of severely alcoholic men during acute intoxication. Lancet 1983;ii:534-537.

12 Greter J, Lindstedt S, Seeman H, Steen G. 2-Hydroxy-2-methylsuccinic acid, a urinary metabolite in propionyl-CoA carboxylase deficiency. Clin Chim Acta 1980;106:103-106.

13 Casazza JP, Felver ME, Veech RL. The metabolism of acetone in rat. J Biol Chem 1984;259:231-236.

14 Peinado J, Lopez-Soriano FJ, Argilés JM. The levels of 2,3-butanediol in acetone-injected rats: effect of pregnancy and fasting. Biochem Arch 1986;2:159-163.

15 McCabe ERB, Goodman SI, Fennessey PV, Miles BS, Wall M, Silverman A. Glutaric, 3-hydroxypropionic, and lactic aciduria with metabolic acidemia, following extensive small bowell resection. Biochem Med 1982;28:229-236.

16 Argilés JM. Has acetone a role in the conversion of fat to carbohydrate in mammals? Trends Biochem Sci 1986;11:61-63. 